\title{
Efifects of DNA Methylation on Cancer and Aging
}

\author{
Simge Sevinç ${ }^{1}\left(\mathbb{D}\right.$, Oytun Erbaş, ${ }^{1,2}(\mathbb{0}$
}

\section{DEOXYRIBONUCLEIC ACID (DNA) METHYLATION}

Deoxyribonucleic acid methylation, an epigenetic mechanism involving the transfer of cytosine from a methyl group to the C5 position to form 5-methylcytosine, which regulates gene expression through collecting proteins involved in gene suppression or inhibiting the binding of transcription factors to DNA. ${ }^{[1]}$ Since it causes differentiation in gene expression, it can give us information in terms of causing hereditary diseases, and also its relation with cancer and aging has been examined. Cancer can be defined as the continuous division of the cell without differentiation through the abnormalities that occur in the cell cycle. Aging is a normal biological process between birth and death in every living organism. Physical aging can cause loss or decrease of functions seen in the whole body. Cellular aging results in cell-based aging and death as a result of all these complicated biological processes.

Deoxyribonucleic acid methylation; It mostly occurs in regions of the genome known as CpG islands. CpG islands are located in the promoter regions at the $5^{\prime}$ end of the genes. It is formed by methyl bonding to the

'ERBAS Institute of Experimental Medicine, Illinois, USA \& Gebze, Turkey ${ }^{2}$ Department of Physiology, Medical Faculty of Demiroğlu Bilim University, Istanbul, Turkey

Correspondence: Simge Sevinç. Deneysel Tıp Enstitüsü, 41470 GebzeKocaeli, Türkiye.

E-mail: simgessevinc@gmail.com

Cite this article as: Sevinç S, Erbaş O. Effects of DNA Methylation on Cancer and Aging. JEB Med Sci 2020;1(3):126-130.

doi: $10.5606 /$ jebms.2020.75627

Received : October 02, 2020

Accepted : October 26, 2020

Published online : December 29, 2020

@2020 Journal of Experimental and Basic Medical Sciences. All rights reserved.

\begin{abstract}
Deoxyribonucleic acid (DNA) methylation; It is an epigenetic factor that occurs via DNA methyltransferases (DNMT) and is often formed by methylation of regions called cytosine phospho guanine $(\mathrm{CpG})$ islands in the genome. It has many macro and micro-level functions such as gene silencing, tissue-specific gene expression, genomic imprinting and the most well-known example, $\mathrm{X}$ chromosome inactivation. Recent evidence has shown that DNA methylation is associated with cancer and aging. It is known that global hypomethylation in cancer occurs directly as a result of an abnormality in DNA methylation. In this review, it was mentioned that abnormal DNA methylation, which also occurs in aging, can be used as a biomarker.

Keywords: Aging, cancer, cytosine phospho guanine, deoxyribonucleic acid methylation.
\end{abstract}

$5^{\text {th }}$ carbon of the cytosine nucleotide after the guanine nucleotide. This epigenetic event that occurs via DNA methyltransferases (DNMT) also causes differentiation in gene expression. DNA methylation is one of the most extensively studied epigenetic modifications in mammals. It provides proper regulation of gene expression or silencing of the gene in normal cells. ${ }^{[2]}$ For example, epigenetic modification is required to silence retroviral elements, regulate tissue-specific gene expression, genomic imprinting, and $\mathrm{X}$ chromosome inactivation. ${ }^{[1]}$ It is one of the best known examples where DNA methylation is required for long-term silencing of a locus in $\mathrm{X}$ chromosome inactivation. ${ }^{[3]}$

In the mechanisms that regulate gene expression, ribonucleic acid (RNA) derivatives called micro RNA (miRNA) are used. The mature miRNA controls gene expression through blocking the translation of the mRNA to which is attached or activating the degradation of the target mRNA. As a result of studies performed with miRNAs, it was observed that DNA methylation regulates miRNA expression. Furthermore, miRNAs can regulate histone 
modifications and DNMT expression as well as DNA methylation while doing so. ${ }^{[1]}$ Although the exact mechanism and source of the abnormal methylation or the triggering factor that initiates this process is unknown, one possibility is that it occurs as a function of aging. ${ }^{[4]}$ Low-level de novo methylation of CpG islands is known to occur in normal tissues and has been shown to increase with age. ${ }^{[4-6]}$ It has been observed in studies conducted in cancer cells that the expression of miRNAs differs greatly from the change that occurs as a result of DNA hypomethylation or DNA hypermethylation. Abnormal methylation that occurs in $\mathrm{CpG}$ islands is also an important distinguishing feature of cancer. Thus, it has been shown that DNA methylation regulatory genes can induce or suppress cancer growth in any specific cancer type..$^{[7]}$

\section{CANCER}

It is a disease caused by altered gene expression that occurs as a result of the normal life cycle and mechanism in cells, and environmental factors trigger genetic changes, and it is a disease with abnormal proliferation. Nowadays, cancer has many types as well as differences in the course of the disease. It can be divided into two groups, depending on whether it undergoes metastasis before specifying it with the tissue or organ it is seen. First of all, there are benign tumors that do not undergo metastasis, which are not life-threatening, and malignant tumors that can metastasize, invade surrounding tissue and can be life-threatening. Tumors are named according to the cells from which they originate with histopathological diagnosis. These; can be classified as carcinomas (originating from epithelial cells), sarcomas (originating from muscle, bone, cartilage and fibrous tissue) and lymphoma-leukemias (originating from the blood and immune system). ${ }^{[8]}$

\section{THE MECHANISM OF CANCER}

Normal cells reproduce until they reach a certain density when examined in in-vitro culture medium and then they stop dividing by entering the G0 phase. This situation is different in cancer cells. Cancer cells that are not affected by cell density-dependent inhibition are not affected by the signal that puts normal cells into the GO phase, so continuous proliferation is observed. The growth of some cell types in culture is dependent on growth factors, but cancer cells require less growth factors and therefore the tumor cell proliferates uncontrolled in both in vivo and in vitro conditions. It is not limited to the tissue or organ where it is formed, but can be transferred to other tissues and organs by using blood or lymphatic pathways called metastases.

Cancer formation occurs as a result of damage accumulated in genes. As well as having a damaged gene from birth, DNA damage caused by viruses, radiation and chemicals that trigger cancer development, called carcinogenic, can also cause cancers as a result of genetic and epigenetic changes. Before looking at the relationship between DNA methylation and cancer, the mechanism and causes of cancer must first be understood. ${ }^{[8]}$

\section{Cancers Caused by Mutation of Human Genes}

There are two groups of genes that mutate and then cause cancer in a healthy cell. The first of these are proto-oncogenes. Proto-oncogenes are known to provide cell growth and proliferation by mostly taking part in signal transmission in the cell. However, these genes are mutated or activated by viruses and turn into genes that have lost their control, called oncogenes, that can lead to cancer development. With this transformation, proto-oncogene genes that are normally; By gaining functions such as gain of function, continuous synthesis and excess synthesis, it creates oncogenes.

The second of the human cancer-causing genes are tumor suppressor genes. Under normal conditions, it prevents cell proliferation and tumor growth. If the loss of function occurs as a result of the damage, uncontrolled growth occurs. They must be deleted or inactivated to form tumors. In cancer development, the inactivation of tumor suppressor genes is an important factor as well as the activation of proto-oncogenes to form oncogenes. ${ }^{[8]}$

\section{Cancers Caused by Viruses}

Since viruses are essential intracellular parasites, they lack a mechanism that produces the essential chemicals necessary for their survival. Viruses cause cancer by activating proto-oncogenes and forming oncogenes. Known viruses and the types of cancer they cause are as follows: Hepatitis B and $C$ viruses cause liver cancer, papilloma viruses cause cervical and other anogenital cancers, epstein-barr virus cause burkitt lymphoma and nasopharyngeal cancers, and T-cell lymphotrophic virus causes adult $\mathrm{T}$ lymphocyte leukemia. ${ }^{[8]}$

\section{Cancer Formation with Carcinogens}

Cancer-causing agents are called carcinogens. Carcinogens are also mutagens. However, it has been found that some carcinogens can trigger cancer 
formation by increasing the activity and synthesis of proteins at the control point of the cell cycle without creating mutations in DNA. Similarly, there are carcinogens in the cell that act like growth factors. Radiation and chemical carcinogens perform their effects by causing DNA damage and mutation. The most important carcinogen in human skin cancer development is UV rays. In addition, carcinogenic chemicals in tobacco smoke are among the main causes of lung cancer. It has been observed that DNA methylation changes that occur with exposure to smoke may play a role in lung cancer etiology ${ }^{[9]}$

\section{AGING}

Aging is a complex, multifactorial biological process shared by all living organisms. ${ }^{[10]}$ In some biological pathways, functional decline can be defined as a single causal way of decline in resting metabolism, while in other pathways the extent of decline is rather broad and elusive, such as the decreased stability of epigenetic models. Factors affecting aging determine the quality and duration of human life. ${ }^{[1]}$

\section{EFFECTIVE FACTORS ON AGING}

There is a lot of evidence that genetic factors are effective in aging. Saccharomycescerevisiae (baker's yeast), Caenorhabditiselegans (nematodes) and Drosophilamelanogaster (fruit fly) are frequently used organisms in genetics studies for aging and many genetic mechanisms for genetic control of life span have been found in studies on these organisms. ${ }^{[12]}$ When Leonard Guarente and his colleagues, a professor at the Massachusetts Institute of Technology, isolated long-living yeast cells, they discovered "sirtuins", a group of genes that enable the yeast cell to survive for a long time. SIR-2 (Silent Information Regulator-2) gene, which is a member of this family, especially has the function of preventing aging. When they took a normal yeast cell and changed the SIR-2 gene, the yeast's lifespan was $50 \%$ longer. This suggests that long-life genes may exist. ${ }^{[13]}$

Another factor is telomere-telomerase activity. Telomere is a specialized nucleoprotein structure found in eukaryotic chromosomes. Telomeres protect the ends of chromosomes from random double strand DNA breaks, protecting the chromosomes from unwanted fusion of chromosome ends and nucleolytic fragmentation. Apart from this physical protection of chromosomes, eukaryotic telomeres have important cellular functions such as chromatin organization, replication of chromosomes and cell proliferation. Therefore, non-functional telomeres also play a role in aging and cancer.

After each cell division, telomeres shorten in length, and after reaching a critical shortness, it is seen that they trigger the mechanisms related to aging. In a study, the telomere length was measured by examining the DNA samples of 143 people. In this study, it was found that those with shorter telomere were 3 times more likely to die from heart disease. Again, in the same study, it was found that those with shorter telomere were 8 times more likely to die from infectious diseases. ${ }^{[14]}$ Telomeres undergo a shortening of about 100 bases in each cell division. Therefore, telomeric DNA must be protected. Telomeres are synthesized by a special enzyme telomerase and are protected from being shortened during each division. Telomerase is an RNA structured reverse transcriptase enzyme. This enzyme are found in embryonic stem cells, unicellular eukaryotes, germ cells and cancer cells. The telomerase enzyme, which is not present in normal somatic cells or is very rare, is active in adult stem cells and embryonic cells. It has been observed that it can be reactivated in cancer cells.

Epigenetic regulations have an effect on aging. If changes in the epigenome can be observed and analyzed for life, human life can become long and healthy by slowing down and accelerating or stopping and directing changes within these observations.

The somatic mutation theory has shown that many diseases occur as a result of mutations accumulated in somatic cells throughout human life. It has been defined that the risk of cancer incidence increases with the accumulation of oncogenic mutations in somatic cells. In addition, in the somatic mutation theory, cellular response capacity to DNA damage has been found to be an important determinant in aging. The response to DNA damage consists of the detection and repair of DNA damage and the control of the cell cycle by apoptosis. ${ }^{[15]}$

Premature aging (progeria) syndromes are another important factor in the genetic control of segmental aging caused by damage to the DNA repair mechanism. Many studies have shown that cellular changes in premature aging syndromes are similar to those in the normal aging process.

Other factors affecting aging can be considered as free radicals (oxidative stress) and environmental factors. The difference in DNA methylation affected 
by these factors may have value as a biological marker of healthy aging versus unhealthy aging and disease risk.

\section{EFFECT OF DNA METHYLATION ON CANCER AND AGING}

Deoxyribonucleic acid hypermethylation was observed in the Polycomb group protein (PcG) target genes as a result of analysis on cancer and aging. It has been reported that global DNA hypomethylation, which is a potential factor in decreasing genome stability and increasing chromosomal abnormalities, has been observed in most types of cancer. ${ }^{[16,17]}$ Dynamic changes in DNA methylation have also been shown to play a key role in pancreatic cancer; therefore, DNA methylation has provided valuable information to better understand the initiation and progression of pancreatic cancer.

A study was conducted using DNA methylation, gene expression, copy number, mutational and clinical data from pancreatic patients. DNA methylation between normal and tumor samples and different gene expression profiles were investigated independently, and methylation levels were correlated with gene expression patterns. A total of $\sim 23,000$ differently methylated $\mathrm{CpG}$ regions $(\Delta \beta \geq 0.1)$ were observed between normal and tumor samples, where most of the $\mathrm{CpG}$ regions are hypermethylated in pancreatic cancer and this is more pronounced in the 5'UTRs and promoter regions compared to gene bodies. ${ }^{[18]}$ Again, abnormal DNA methylation in the promoter regions of the gene that leads to inactivation of tumor suppressor and other cancerrelated genes in cancer cells is the best defined epigenetic event in gastric cancer. ${ }^{[19]}$ Age-related CpG DNA methylation changes in human blood, brain, kidney and skeletal muscle tissue are also among the identified examples. Interestingly, hypomethylated CpG regions were found to be more strongly associated with tissue-specific, age-related changes than hypermethylated $\mathrm{CpG}$ regions. ${ }^{[20]}$

Some of the methylation changes that occur with age, appear in certain regions of the genome. This shows us that at least some of the DNA methylation changes during aging are not accidental, but may be related to biological mechanisms involved in the aging process. Apart from life span, DNA methylation also differs between organisms with the same genetic makeup, such as queen and worker honey bees, when exposed to environmental stimuli, and this is an altered gene expression pattern. General DNA hypomethylation that occurs during aging also occurs in specific gene promoters, including age-related progressive loss of DNA methylation, ITGAL and IL17RC. ${ }^{[21,22]}$ Concurrent with general and localized DNA hypomethylation during aging, hypermethylation has been predicted to occur in specific $\mathrm{CpG}$ regions of the genome to suppress the expression of specific genes. ${ }^{[10,22-24]}$ As a result of all these experiments and analyzes, the effect of DNA methylation on cancer and aging was mentioned. It has also been mentioned that hypomethylation, especially in DNA, can be used as a biomarker in both some types of cancer and aging.

\section{Conclusion}

As mentioned, DNA methylation has extremely important effects on cancer and aging. This review, which was made under the name of associating these two popular terms with DNA methylation, aims to shed light on the cause and effect between these three topics. However, increasing evidence suggests that most methylation changes occur in a programmed manner and occur in a subpopulation of tissue cells during normal aging, possibly making them susceptible to tumorigenesis. As a result of all these analyzes, it has been revealed that what a vital function DNA methylation has. Although it was not possible to determine and analyze DNA methylation on a genome-wide basis in terms of technology, today the studies have been expanded and verified with the development of new generation sequencing technologies. Thanks to such possibilities, with more experiments and more up-to-date information, abnormalities in methylation will now become as easy and commonplace as diagnosing it by taking an $x$-ray. In this way, the information we have obtained will play an important role in finding a treatment for a disease that is currently dreamed of and that reduces our quality of life, such as delaying aging or stopping cancer.

\section{Declaration of conflicting interests}

The authors declared no conflicts of interest with respect to the authorship and/or publication of this article.

\section{Funding}

The authors received no financial support for the research and/or authorship of this article.

\section{REFERENCES}

1. Moore LD, Le T, Fan G. DNA methylation and its basic function. Neuropsychopharmacology 2013;38:23-38. 
2. Kulis M, Esteller M. DNA methylation and cancer. Adv Genet 2010;70:27-56.

3. Riggs AD, Pfeifer GP. X-chromosome inactivation and cell memory. Trends Genet 1992;8:169-74.

4. Issa JP. Aging and epigenetic drift: a vicious cycle. J Clin Invest 2014;124:24-9.

5. Kulis $M$, Merkel A, Heath $S$, Queirós AC, Schuyler RP, Castellano $G$, et al. Whole-genome fingerprint of the DNA methylome during human B cell differentiation. Nat Genet 2015;47:746-56.

6. Teschendorff $A E$, Menon $U$, Gentry-Maharaj $A$, Ramus SJ, Weisenberger DJ, Shen $\mathrm{H}$, et al. Age-dependent DNA methylation of genes that are suppressed in stem cells is a hallmark of cancer. Genome Res 2010;20:440-6.

7. Karimzadeh MR, Pourdavoud P, Ehtesham N, Qadbeigi $M$, Asl MM, Alani B, et al. Regulation of DNA methylation machinery by epi-miRNAs in human cancer: emerging new targets in cancer therapy. Cancer Gene Ther 2020 [Epub ahead of print]

8. Topaktaş M. Genetik. 1. Baskı. Ankara. Nobel Yayınevi; 2014.

9. Battram T, Richmond RC, Baglietto L, Haycock PC, Perduca V, Bojesen SE, et al. Appraising the causal relevance of DNA methylation for risk of lung cancer. Int J Epidemiol 2019;48:1493-504.

10. Pal S, Tyler JK. Epigenetics and aging. Sci Adv 2016;2:e1600584.

11. Jung $M$, Pfeifer GP. Aging and DNA methylation. BMC Biol 2015;13:7.

12. Bishop NA, Guarente L. Genetic links between diet and lifespan: shared mechanisms from yeast to humans. Nat Rev Genet 2007;8:835-44.

13. Guarente L, Kenyon C. Genetic pathways that regulate ageing in model organisms. Nature 2000;408:255-62.

14. Cawthon RM, Smith KR, O'Brien E, Sivatchenko A, Kerber
RA. Association between telomere length in blood and mortality in people aged 60 years or older. Lancet 2003;361:393-5.

15. Al-Wahiby S, Wong HP, Slijepcevic P. Shortened telomeres in murine scid cells expressing mutant hRAD54 coincide with reduction in recombination at telomeres. Mutat Res 2005;578:134-42.

16. Wilson AS, Power BE, Molloy PL. DNA hypomethylation and human diseases. Biochim Biophys Acta 2007;1775:138-62.

17. Berman BP, Weisenberger DJ, Aman JF, Hinoue T, Ramjan $Z$, Liu Y, et al. Regions of focal DNA hypermethylation and long-range hypomethylation in colorectal cancer coincide with nuclear lamina-associated domains. Nat Genet 2011;44:40-6.

18. Mishra NK, Guda C. Genome-wide DNA methylation analysis reveals molecular subtypes of pancreatic cancer. Oncotarget 2017;8:28990-9012.

19. Qu Y, Dang S, Hou P. Gene methylation in gastric cancer. Clin Chim Acta 2013;424:53-65.

20. Day K, Waite LL, Thalacker-Mercer A, West A, Bamman MM, Brooks JD, et al. Differential DNA methylation with age displays both common and dynamic features across human tissues that are influenced by CpG landscape. Genome Biol 2013;14:R102.

21. Wei L, Liu B, Tuo J, Shen D, Chen P, Li Z, et al. Hypomethylation of the IL17RC promoter associates with age-related macular degeneration. Cell Rep 2012;2:1151-8.

22. Cedar H, Bergman Y. Programming of DNA methylation patterns. Annu Rev Biochem 2012;81:97-117.

23. Weidner $\mathrm{Cl}$, Wagner $\mathrm{W}$. The epigenetic tracks of aging. Biol Chem 2014;395:1307-14.

24. Horvath S. DNA methylation age of human tissues and cell types. Genome Biol 2013;14:R115. 